\title{
Iniciación a la Práctica Profesional: la mirada reflexiva de los estudiantes de Educación Básica
}

\author{
Maria de Lurdes Carvalho \\ Instituto de Educação, Universidade do Minho, Portugal
}

\begin{abstract}
Resumo
El Practicum se constituí como el pasaporte para la vida laboral en que cada estudiante va edificando su trayecto profesional movilizando saberes, confrontando expectativas, valores y ética, construyendo de forma compartida su identidad profesional. Se trata de un estudio de caso centrado en las narrativas de los estudiantes desarrolladas en la asignatura de Practicum y su objetivo es averiguar sus percepciones cerca de los trayectos formativos construidos en el ámbito de la referida asignatura. La adquisición del conocimiento (científico, metodológico, pedagógico), articulación teoría y práctica, e interdisciplinariedad asumen relevancia en los resultados como fuente de crecimiento personal y profesional.

Palabras-clave: Formación inicial de Profesores; Identidad Profesional; Conocimiento Profesional; Práctica Profesional.
\end{abstract}

\section{Introdução}

El Practicum en la formación inicial de profesores de Educación Infantil y Primaria representa un tiempo y un espacio formativo especifico, que bajo la circularidad de saberes (Burguiere, 2002), saberes acerca (Day, 2005), del y en el trabajo (Tardif, 2004), faculta la emergencia del proceso de construcción, reconstrucción, co-construcción de la identidad profesional, necesario a la adaptación a la acción laboral. Pero, "el conocimiento no se ubica en parcelas inconexas, hay que crear espacios que permitan conectar contenidos" (Navarro Soria, González Gómez, López Monsalve, \& Botella Pérez, 2015, p. 101). Es entonces necesario asegurar que los centros de prácticas no sean "un mosaico de miembros procedentes de varias disciplinas" (ibídem), y que los dos contextos formativos caminen juntos en coherencia, centralidad, complementariedad (Zabalza, 2013) y reciprocidad.

El Practicum suele representar una inestimable experiencia personal de aprendizaje pero exige una acción tutorial correcta (Cid Sabucedo \& Ocampo Gómez, 2006), interdisciplinar, próxima y continúa, capaz de crear momentos de reflexión, de aprendizaje participativa, conjunta y colaborativa, o sea, que se asuma como un proceso de ayuda y orientación continúa, dinámica e integradora en un proceso de aprendizaje, conocimiento e identidad profesionales.

Asimismo, el rol del tutor universitario es acompañar, orientar y apoyar al estudiante a construir sus conocimientos y creencias de la profesión ser maestro que, a través de un proceso de "mediación, canalización, interrelación y facilitación de distintos procesos de transformación y/o cambio social a lo largo de la vida" (Martínez Clares, 2011, p. 47), puede crear tiempos de conceptualización, clarificación, formalización, reflexión y movilización de conocimientos, nuevos o ya adquiridos por el estudiante a lo largo de su formación (académica y práctica) y de su historia de vida. Tutores universitarios y estudiantes enfrentan juntos una práctica reflexiva caracterizada por el principio de dialogicidad como en una relación de comunicación entre iguales (Navarro Soria, González Gómez, López Monsalve, \& Botella Pérez, 2015).

A la vez, el tutor de centro - maestro-tutor - asume un papel central en esta formación a través de procesos de demonstración, análisis e cuestionamiento de y sobre la acción pedagógica en un contexto real y objetivo. En un tiempo real, la acción pedagógica está siendo compartida entre maestro-tutor $\mathrm{y}$ estudiante permitiendo una reflexión acerca de, de la y en la acción más profunda, creándose una comunidad de prácticas y de aprendizaje profesional.

La acción tutorial no se hace sin el estudiante. La acción tutorial asume una dimensión auto reflexiva y auto formativa en que los estudiantes, futuros profesores, empiezan a adquirir confianza y motivación por su conocimiento profesional, desarrollan su capacidad de hacer oír su voz como investigadores de su propia práctica y constructores del saber profesional especifico inherente a su función laboral (Alarcão \& Roldão, 2008).

El Prácticum es así un tiempo y espacio dónde los estudiantes tienen la oportunidad de sumergirse en la profesión, desarrollando su profesionalidad docente, observando la articulación entre la teoría y práctica como una teoría práctica (Alarcão \& Roldão, 2008) en un contexto real de actividad laboral, desarrollando su "inteligencia pedagógica, multidimensional y estratégica [y su] capacidad reflexiva y auto reguladora" (Alarcão \& Roldão, 2008, p. 16), elaborando proyectos de aprendizaje, descubriendo y adquiriendo su propia identidad profesional, testando sus competencias, en suma, apropiándose del conocimiento profesional que fueron adquiriendo (Carvalho, 2013). En esta inmersión laboral enfrentan la realidad educacional concreta (con sus dificultades, problemas, potencialidades) a través de interacciones con1@s niñ@s, de investigaciones y desarrollo de propuestas de acción nuevas e innovadoras en consonancia con el contexto social y cultural en que se encuentran. De esta forma, y gradualmente, los estudiantes adquieren y desarrollan competencias personales y profesionales, movilizando de forma interrelacionada conocimientos, actitudes y procedimientos que dependen, simultáneamente, del empeño personal y de la existencia de un contexto favorable (Leitão \& Alarcão, 2006). 
Así, todos los intervinientes, conjuntamente participes de este proceso de formación, con roles diferenciados pero mutuamente dependientes y articulados, permiten el suceso de la formación de futuros profesionales emancipados, reflexivos y transformadores de la educación y sociedad de mañana. $\mathrm{O}$ sea, todo el equipo (tutores universitarios, maestros-tutores y estudiantes, se instituyen como elementos clave del Prácticum, que con sus funciones diferenciadas, constituyen comunidades profesionales de aprendizaje que, integrando solo personas de una misma familia profesional (Tejada Fernández, 2009) buscan el crecimiento profesional (Molina Ruiz, 2005) y la mejora de calidad (Tejada \& Carvalho, 2013, p. 3).

El estudiante asume un rol central en todo el proceso formativo pero también en el proceso evaluativo. En su evaluación, el estudiante cruza procesos de supervisión, de transformación y construcción identitária a través de procesos de: i) auto supervisión en sus autoevaluaciones; ii) supervisión vertical con evaluaciones dialogadas con tutores universitarios y de centros, iii) supervisión horizontal entre pares que hacen Practicum en la misma escuela y/o otros estudiantes del mismo grupo de supervisión y/o clase (Alarcão \& Roldão, 2008; Castejón, López, Julián, \& Zaragoza, 2011; Fraile, López, Castejón, \& Romero, 2013; Vallés, Ureña, \& Ruiz, 2011. La implicación en la práctica evaluativa ayuda los estudiantes a desarrollar su pensamiento superior, más allá de sus capacidades personales y profesionales de reflexión, responsabilidad, autonomía, criticidad y participación en su propio proceso de formación profesional (Rodríguez \& Herrera, 2009).

\section{Metodología}

La titulación en Educación Básica tiene como objetivo principal la formación de diplomados para que puedan proseguir estudios que les permite el acceso a la docencia en el ámbito de la educación infantil y de la educación primaria $\left(1^{\circ}\right.$ e $2^{\circ}$ Ciclo), contemplando también la posibilidad de conferir habilitación para el desempeño de funciones como técnico en educación básica con tareas de apoyo y colaboración en actividades curriculares, extracurriculares y de complemento curricular.

Inserida en la componente de Iniciación a la Práctica Profesional (Practicum) la asignatura de Proyectos Interdisciplinares de Investigación y Acción Pedagógica (PIIAP) se desarrolla en el $3^{\circ}$ año de la titulación de Educación Básica ( $1^{\circ}$ ciclo de estudios) en el Instituto de Educación, Universidad del Miño (Portugal). En esta asignatura se procura garantizar el contacto y formación de los estudiantes en diferentes perspectivas y problemas educativos $\left(1^{\circ}\right.$ e $2^{\circ}$ ciclo de educación infantil, escuela primaria, escuela secundaria) que envuelven niños y los profesionales. A través de una intervención, de carácter investigativa, en el contexto de educación formal se pretende que, en articulación con las restantes asignaturas del plan de formación, la acción pedagógica en el contexto real y concreto resulte en aprendizajes y en desarrollo de competencias profesionales. Aún más, se pretende desarrollar aprendizajes de aptitudes y prácticas deontológicamente balizadas a través de reflexiones sobre la dimensión ética inherente a su acción profesional.

PIIAP se desarrolla en fases que permiten la inmersión progresiva en el contexto, lo que presupone la existencia de alternancia entre la universidad y el centro de prácticas. Así, en la universidad se oscila entre sesiones de formación y reflexión en tutorías conjuntas e individuales, seminarios temáticos con expertos (internos y externos) y sesiones teórico-prácticas. Por su turno, y en alternancia, ocurren las sesiones de intervención en los centros y respectiva acción tutorial con los maestros tutores. Esta metodología de inmersión inicia en el primero semestre del año lectivo con una fase de observación participante en los centros que permite un conocimiento próximo y más profundo de los contextos de intervención (contextos, escuela, aula, nin@s). Posteriormente, en el segundo semestre se desarrolla la etapa de intervención (construcción, desarrollo, evaluación de un proyecto).

Los dispositivos de formación son distintos y complementares de forma a poder conducir el proceso evolutivo, formativo, reflexivo de cada estudiante. Así, la evaluación se centra especialmente en i) la acción tutorial apoyada y supervisada por los tutores (universitarios y maestro-tutor) en que se debate la investigación, construcción, desarrollo y evaluación de un proyecto de intervención; ii) portafolio de aprendizaje donde cada estudiante organiza el trabajo realizado en y con el centro y su análisis reflexiva de crecimiento profesional; iii) las autorreflexiones o narrativas, en cuanto memoranduns de aprendizaje donde cada estudiante reflexiona globalmente sobre sus aprendizajes y conocimientos teóricos y prácticos y su movilización capaz de le permitir alcanzar un nivel de pensamiento superior.

En este estudio se ha optado por el paradigma cualitativo, fundamentalmente de carácter descriptivointerpretativo (Cohen \& Manion, 1990) naturalista, humanista o etnográfico (Albert Gómez, 2007), puesto que pretende centrarse en la interpretación y comprensión de los fenómenos a partir de los significados e intenciones de las personas implicadas en el contexto educativo (Latorre, Del Rincón, \& Arnal, Bases metodológicas de la investigación educativa, 2003).

El objetivo es pues percibir las voces de los estudiantes buscando una mirada interna de los informantes sobre sus trayectos construidos manifiestos en sus narrativas realizadas en el ámbito de la asignatura de Practicum, considerando:

- Aprendizajes realizados y dificultades vividas;

- Puntos fuertes de las sesiones de trabajo de campo en los centros;

- Contribución de las diferentes asignaturas del grado en la construcción y desarrollo del proyecto de intervención;

- Importancia de esta experiencia de iniciación a la práctica profesional;

- Reflexión crítica de la contribución de la asignatura para la formación profesional - seminarios, trabajo de campo, sesiones teórico-prácticas, supervisión $\mathrm{y}$ tutorías, ... 
La muestra de este estudio se ha conformado a través de un muestreo intencional o de conveniencia (Tójar, 2006) de estudiantes $(\mathrm{n}=77)$ que en 2011-12 cursaran asignatura de Proyectos Interdisciplinares de Investigación y Acción Pedagógica II (PIIAP II) de la titulación de Educación Básica del Instituto de Educación de la Universidad do Miño.

La recogida de datos se realizó entonces en las autorreflexiones y posteriormente hecha el análisis de contenido considerando tres dimensiones:

- Trayecto epistemológico - indicadores relativos a la construcción y apropiación de conocimientos y saberes de diferentes áreas (científicas, profesionales, teóricas, reflexivas, metodológicas/ pedagógica) relacionadas con la profesión docente, la acción educativa, la realidad social y cultural del contexto;

- Trayecto de praxiológico - indicadores referentes al desarrollo de la pericia en la acción reflexiva considerando la relevancia del contexto educativo, desarrollo profesional (competencias, experiencias, aprendizajes y formación académica) y desarrollo personal (aprendizajes y motivaciones), así como conocimiento y saberes teóricos y prácticos capaces de una formación profesional integradora;

- Trayecto identitário - indicadores inherentes a la construcción de la identidad profesional, que siendo singular e idiosincrática, es influenciada por procesos interactivos. En este sentido, considerase en esta dimensión los aspectos relacionados con las relaciones intrapersonales e interpersonales con los tutores (universitarios y maestros tutores) en el ejercicio de su práctica docente que se pautan por la ética, el respecto y la valorización de las diversidades.

El tratamiento estadístico aplicado a los datos fue un análisis descriptivo-interpretativo apoyado por el software MAXQDA 10.

\section{Presentación y discusión de resultados}

Para dar respuesta al objetivo de esta investigación fueran analizadas las autorreflexiones/narrativas elaboradas por los estudiantes al final de la titulación y consideradas las dimensiones ya mencionadas: Trayecto epistemológico; Trayecto praxiológico; Trayecto identitario. Todavía, posteriormente fue integrada una cuarta dimensión "Miradas" de la titulación.

Globalmente, los estudiantes enfatizan sus trayectos praxiológicos coincidiendo con algunos estudios ya presentes en la bibliografía científica (e.g. Alarcão \& Roldão, 2008; Zabalza, 2013). Por otras palabras, el énfasis de los trayectos de formación construidos a lo largo de la titulación recae en los sus trayectos de (re)construcción de los aprendizajes, conocimientos, experiencias y competencias profesionales (55\%). Los trayectos de emergencia de la identidad profesional (35\%) están también muy presentes y vinculados a los tutores (universitarios y de centro) y al grupo de niñ@s de las escuelas en que desarrollaran sus prácticas. Los trayectos epistemológicos (10\%) emergen como "pasos" para adquirir conocimientos profesionales teóricos adquiridos en contexto de la universidad (Figura 1).

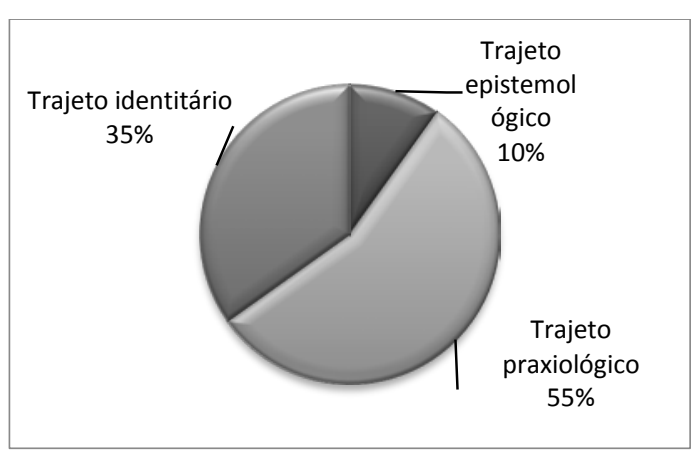

Figura 1 - Distribución de los trayectos profesionales

Considerando el trayecto epistemológico, los conocimientos metodológicos/ pedagógicos (87\%) de diferentes asignaturas asumen relevancia para el desarrollo del Practicum, así como el conjunto de conocimientos teóricos (48\%), profesionales (37\%) y reflexivos (2\%). Afirman "Con base en lo que aprendí a lo largo de la titulación, fue posible crear una multiposibilidad de formas de exploración de los diversos contenidos curriculares (A01). A su vez "en el decurso de todo el proceso pude verificar cuanto fueran importantes todas las asignaturas, y en muchos momentos prácticos pude recordar situaciones que fueran abordadas y trabajadas en clases teóricas del grado, funcionado como apoyo al desarrollo de las actividades propuestas" (A01). O sea, en Practicum fue posible verificar "la importancia que las otras unidades curriculares han tenido en el desarrollo de este proyecto, (...) y nuestra actividad tenía el objetivo de ser interdisciplinar" (A03), y además facilitó espacio para hacer propuestas educativas, para "colocar en práctica, lo que aprendemos en la teoría" (A03). Como que evaluando la formación académica al final del trayecto en la universidad, podemos constatar que "todas las unidades curriculares del grado, sirvieran de base en mi desempeño en el contexto y también para el desarrollo y construcción del proyecto, ya que se trataba de una intervención en que se enfatizaba la interdisciplinaridad. Por eso, todas las unidades curriculares fueran esenciales para un proyecto más rico y completo" (A06, 2-2)

Los trayectos praxiológicos, que enfatizan la (re)construcción de los aprendizajes, conocimientos, experiencias y competencias profesionales, surgen en las narrativas como una referenciación a la acción, en la adquisición y desarrollo de competencias pedagógicas (61\%), investigativas (14\%) y reflexivas $(25 \%)$. El tiempo inicial de observación es "fundamental, para la formación profesional docente, porque posibilita la construcción de un conocimiento profesional, con significado profesional e personal, permitiendo la movilización y articulación de conocimientos y procedimientos, de las diferentes áreas del saber aprehendidas a lo largo de la licenciatura, de las creencias e ideologías, así como de experiencias (A34).

Los estudiantes enfatizan el contacto con los centros, con los profesores, con1@s niñ@s, con "los artefactos" 
que ven los tutores utilizar en su acción pedagógica, y aún enfatizan sus intervenciones didáctico-educativas (Alarcão \& Roldão, 2008, p. 27). La implementación de los proyectos permitió “aprender con los niñ@s, oírlos, dejarlos expresar lo que tenían en mente y esencialmente comprehender las dificultades que poseían y, en conjunto, tentar ultrapasar" (A76). La existencia de un "contexto favorable a la realización de las actividades fue también fundamental, pues tengo perfecta noción de que si el mismo e, consecuentemente, la turma no primasen por ser positivas características la implementación del proyecto podría revelarse más complexa y no llegar a los buenos resultados que pienso ter alcanzado" (A49). Esta narrativa apunta en el sentido de Leitão e Alarcão (2006).

Se verifica, todavía, que hay una tenue referencia al desarrollo de competencias investigativas, muchas veces asociadas al "poco tiempo" disponible. El envolvimiento personal, asociado al aprendizaje en acción, justifica que estas experiencias pre-profesionales en el Practicum, son ejes centrales de formación y asumen especial relevancia (Alarcão \& Roldão, 2008).

Los trayectos identitários relatan la construcción de identidad profesional y la importancia de las relaciones profesionales. Los maestros tutores aparecen con gran relevancia en el proceso así como los niño@s constituyéndose como un referencial identitario nuclear (Alarcão \& Roldão, 2008, p. 27). Se asume una identidad profesional que considere que "l@s niñ@s son seres activos en la construcción de su propio conocimiento [por lo que] es esencial que cada uno de nosotros no se olvide que el profesor es solo un mediador que conduce la línea de pensamiento de los mismos" (A1).

Este tiempo de inmersión en la vida laboral, tiempo de integración en la familia profesional, facilita la integración en una identidad colectiva y el hacer frente a habilidades y dificultades personales, conocer sus propias limitaciones. En este sentido se aprende a que "los incidentes críticos, sirven para nos aproximar cada vez más de la realidad profesional permitiendo así saber cuáles son mis dificultades en cuanto futura educadora de infancia" (A3).

La narrativa de la autorreflexión también constituyó un paso para la construcción de la identidad y que "aprendí a hacer (...) un Profesional de Educación debe reflexionar acerca de sus prácticas, basándose en la experiencia, en la investigación, (...) permitiéndome evaluar mi desempeño profesional (...), constituyendo, la reflexión, un elemento fundamental del proceso de aprendizaje. De este modo, tenté ser una profesional reflexiva, esto es, procuré equilibrar la acción y el pensamiento, originando una nova práctica asiente en la reflexión de la experiencia, de las creencias y valores, reflexión esa que permitió la investigación y la mejora de lo que estaba haciendo, lo que demuestra que enseñar no es solo una arte, más bien una procura incesante con el objetivo de encontrar las condiciones para ocurrir el aprendizaje. A lo largo de las actividades, procedí, varias veces, a la reflexión en la acción... (A76)

Este despertar de la identidad profesional permitió también hacer opciones, tomar decisiones cuanto al futuro profesional porque "percibí como será nuestro día-a-día en una sala de aula, y en mi caso, percibir cual la verdadera vocación: si es educadora de infancia o profesora de primaria, ayudándome así a tomar decisiones para el master a seguir" (A2).

En este proceso de descubierta de su "verdadera vocación" el rol del maestro tutor asume especial relevancia. La apreciación muy positiva del rol del maestro tutor (31\%) va en desfavor del tutor universitario (13\%), confirmando el estudio de Alarcão e Roldão (2008) en que los maestros tutores son globalmente referenciados como ejerciendo una "influencia muy positiva y mucho más relevante" que los tutores universitarios (Alarcão \& Roldão, 2008, p. 27).

En síntesis, estos datos confirman que el Practicum es un tiempo y espacio de gran significado y relevancia para los estudiantes futuros maestros pues proporciona un conjunto de conocimientos profesionales de y en la realidad educativa, la inmersión en su actividad laboral futura (Latorre \& Blanco, 2011), la inserción en su familia profesional (Tejada, 2009) imbricada con una determinada cultura profesional.

La asignatura de Proyectos Interdisciplinares de Investigación y Acción Pedagógica asume especial relevancia y permite la "adquisición de nuevos conocimientos y el perfeccionar aquellos que ya tenía adquirido en otras asignaturas" (A06). Todavía, los estudiantes consideran que el tiempo de inmersión, en duración y periodicidad, es escasos a lo largo de su formación inicial pues constituye una experiencia con el mundo laboral futuro capaz de clarificar el ámbito profesional y crear espacio de toma de decisiones.

\section{Conclusiones}

La Iniciación a la Práctica Profesional en la Educación Básica, incluso las narrativas que los estudiantes hacen como elemento de evaluación, permite una mirada reflexiva acerca, del y en el Practicum que ni siempre hay oportunidad o tiempo de desarrollar en su formación inicial de otras asignaturas.

Esta asignatura, de especial relevancia para los futuros maestros, facilita una pequeña inmersión en la futuro mundo laboral/profesional, que a través de experiencias $\mathrm{y}$ aprendizajes vivenciadas permiten que cada alumno objetive sus capacidades y limitaciones, se supere a sí mismo, haga opciones y tome decisiones, o sea, que desarrolle sus competencias de saber, saber-hacer, saber-ser y saber-estar. En un espacio de proximidad con niñ@s y sus familias y de una familia profesional, cada estudiante, futuro maestro, encuentra su espacio de acción, su identidad profesional, personal y relacional. Si bien que en este estudio los estudiante valoren sus experiencias, la articulación teoría y práctica, el rol de los tutores hay aún un camino a construir de consolidación de competencias. El saber-hacer sobresale las narrativas personales en desfavor del saber, mientras que el saber ser y saber-estar son especialmente nombradas como competencias profesionales y personales, más allá de la profesión. De forma reiterada, es necesario repensar el lugar que el Practicum asume en el plano de estudios, los ECTS que cada universidad destina, la real formación integrada, pensando en la formación en y para el trabajo. 


\section{Referências}

Alarcão, I., \& Roldão, M. (2008). Supervisão: um contexto de desenvolvimento profissional dos professores. Mangualde: Edições Pedago.

Albert Gómez, M. J. (2007). La Investigación Educativa: Claves Teóricas. Madrid: McGraw-Hill Interamericana de España.

Burguiere, E. (2002). Entretien avec Jean-Louis Martinand. Recherche et Formation, 40, pp. 87-94. Obtido

http://ife.ens-lyon.fr/edition-electronique/archives/rec herche-formation/web/fascicule.php?num_fas $=254$

Carvalho, M. (2013). Formación inicial de profesores: sinergias construyendo una nueva realidad. Barcelona: Departamento Pedagogía Aplicada.

Castejón, F., López, V., Julián, J., \& Zaragoza, J. (2011). Evaluación formativa y rendimiento académico en la formación inicial de profesorado de Educación Física. Revista Internacional de Medicina y Ciencias de la Actividad Física y el Deporte, 11(42), pp. 328-346. Obtido de http://www.redalyc.org/articulo.oa?id=54222171007

Cid Sabucedo, A., \& Ocampo Gómez, C. (Mayo-agosto de 2006). Funciones tutoriales en el Prácticum correspondiente al actual plan de estudios de Magisterio en la Universidad de Vigo. Revista de Educación, 340, pp. 445-472. Obtido de http://www.revistaeducacion.mec.es/re340.htm

Cohen, L., \& Manion, L. (1990). Métodos de investigación educativa. Madrid: La Muralla.

Day, C. (2005). Formar Docentes. Cómo, cuándo y en qué condiciones aprende el profesorado. Madrid: Narcea.

Fraile, A., López, V., Castejón, F., \& Romero, E. (2013). La evaluación formativa en docencia universitaria y el rendimiento académico. Aula Abierta, 41(2), pp. 23-34. Obtido de http://dialnet.unirioja.es/servlet/articulo? codigo $=4239$ 063

Latorre, A., \& Blanco, F. (2011). El practicum como espacio de aprendizage profesional para docentes en formación. REDU - Revista de Docencia Universitaria, 9(2), pp. 35-54. Obtido de http://red-u.net/redu/index.php/REDU/issue/view/64

Latorre, A., Del Rincón, D., \& Arnal, J. (2003). Bases metodológicas de la investigación educativa. Barcelona: Experiencia.

Leitão, A., \& Alarcão, I. (2006). Para uma nova cultura profissional: uma abordagem da complexidade na formação inicial de professores de $1 .^{\circ}$ CEB. Revista Portuguesa de Educação. Vol 19(2), 51-84. Obtido de http://www.scielo.gpeari.mctes.pt/scielo.php?script=sc i_arttext\&pid=S0871-91872006000200004

Martínez Clares, P. (2011). La orientación en tiempos de cambio. Em J. J. Maquilón Sánchez, La formación del profesorado en el siglo XXI. Propuestas ante los cambios económicos, sociales y culturales (pp. 45-62). Murcia: Ediciones de la Universidad de Murcia (edit.um).

Molina Ruiz, E. (2005). Creación y deesarrollo de comunidades de aprendizaje: hacia la mejora educativa. Revista de Educación, 337, 235-250. Obtido de http://www.revistaeducacion.mec.es/re337_12.htm

Navarro Soria, I., González Gómez, C., López Monsalve, B., \& Botella Pérez, P. (2015). Aprendizaje de contenidos académicos y desarrollo de competencias profesionales mediante prácticas didácticas centradas en el trabajo cooperativo y relaciones multidisciplinares. Revista de Investigación Educativa, 33(1), $\quad$ pp. 99-117. doi:http://dx.doi.org/10.6018/rie.33.1.183971

Rodríguez, C., \& Herrera, L. (2009). Análisis correlacional-predictivo de la influencia de la asistencia a clase en el rendimiento académico universitario. Estudio de caso en una asignatura. Profesorado. Revista de Curriculum y Formación del Profesorado, 13(2), pp. 1-13. Obtido de http://www.ugr.es/ recfpro/rev132COL4.pdf

Tardif, M. (2004). Los saberes del docente y su desarrollo profesional. Madrid: Narcea.

Tejada Fernández, J. (2009). Competencias docentes. Profesorado. Revista de Currículum y Formación, 13, n. 2, pp. 1-15. Obtido de http://www.ugr.es/ recfpro/rev132COL2.pdf

Tejada, J., \& Carvalho, M. (2013). El Prácticum en la formación inicial de maestros: percepciones de los tutores de universidad de educación infantil y primaria. Em P. Muñoz, M. Raposo-Rivas, M. González, M. Martínez-Figueira, M. Zabalza-Cerdeiriña, \& A. Pérez-Abellás, Un Prácticum para la formación integral de los estudiantes (pp. 1583-1596). Santiago de Compostela: Andavira.

Tójar, J. (2006). Investigación Cualitativa. Comprender y Actuar. Madrid: La Muralla.

Vallés, C., Ureña, N., \& Ruiz, E. (2011). La evaluación formativa en docencia universitaria. Resultados globales de 41 estudios de caso. REDU - Revista de Docencia Universitaria, 9(1), pp. 135-158. Obtido de http://red-u.net/redu/index.php/REDU/issue/view/63

Zabalza, M. (2013). El Practicum como contexto de aprendizajes. Actas XII Symposium Internacional sobre el Practicum y las Prácticas en Empresas en la Formación Universitaria: Un Practicum para la formación integral de los estudiantes (pp. 51-68). Poio (Pontevedra): http://redaberta.usc/poio. Obtido de http://red-u.net/redu/index.php/REDU/issue/view/63 